\title{
Nonconforming Discretization of the PMCHWT Integral Equation Applied to Arbitrarily Shaped Dielectric Objects
}

\author{
Ivan Sekulic $^{1 *}$, Eduard Ubeda ${ }^{1}$ and Juan M. Rius ${ }^{1}$ \\ ${ }^{1}$ AntennaLab, Universitat Politecnica de Catalunya (BarcelonaTECH), Barcelona, Spain, ivan.sekulic@tsc.upc.edu
}

\begin{abstract}
The Poggio-Miller-Chan-Harrington-Wu-Tsai (PMCHWT) integral equation is widely used in the scattering analysis of dielectric bodies. The RWG set is normally adopted to expand the electric and magnetic currents in the Method of Moments (MoM) discretization of the PMCHWT formulation. This set preserves normal continuity across edges in the expansion of currents. However, in the analysis of composite objects, the imposition of such continuity constraint around junctions, where several regions intersect, becomes convoluted. We present a new nonconforming discretization of the PMCHWT formulation so that currents are expanded with no continuity constraint across edges. This becomes well-suited for the analysis of composite objects or nonconformal meshes, where some adjacent facets have no common edges. We show RCS results where the nonconforming PMCHWT implementation, facet-oriented, shows similar or better accuracy as the conventional approach, edge-oriented, for a given degree of meshing.
\end{abstract}

Index Terms-Integral Equations, Method of Moments, PMCHWT formulation

\section{INTRODUCTION}

The conventional MoM-discretization of the PMCHWT integral formulation [1][2][3] usually relies on the low-order RWG set, which preserves the normal continuity of the currents across the edges arising from the meshing of the problem [4]. The hypersingular Kernel contributions arising in the expansion of scalar potentials are then cancelled out. However, in the analysis of a composite object, made up of several regions with different electromagnetic properties, the definition of the RWG set around junctions, edges where several regions intersect, becomes a cumbersome task [5]. Recently, new nonconforming schemes, based on the facetoriented monopolar-RWG set, with no interelement continuity constraints, have been developed for the scattering analysis of conductors with the Electric Field Integral Equation (EFIE) [6][7][8]. In these schemes, the hypersingular Kernel contributions are properly evaluated by testing the fields off the boundary surface, in the inner region of the body, through two different non-Galerkin testing strategies. The fields are either tested over small volumetric entities (tetrahedral elements or wedges) attached to the boundary elements [6][7] or with RWG functions defined over pairs of connected triangles, one matching a surface facet and the other one oriented normally inside the conductor [8]. The latter testing scheme, "tangential- normal", cancels out the hypersingular scalar potential contributions and leads to an easier matrix generation than the former "volumetric" approach. In this paper, we extend these nonconforming schemes to the scattering analysis of single dielectric bodies with the PMCHWT integral equation. Since the PMCHWT formulation now involves two equivalent problems, the testing of the fields needs to be conducted in the region where, in light of the equivalence theorem, the fields have to be zero. In this paper, we show with RCS results that the nonconforming PMCHWT implementation shows similar or better accuracy as the conventional divergence-conforming approach for a given degree of meshing. The nonconforming PMCHWT implementation, facet-oriented, appears as a promising tool for the robust and flexible scattering analysis of piecewise homogeneous objects.

\section{NONCONFORMING DiSCRETIZATION OF THE PMCHWT FORMULATION}

The expansion of the electric and magnetic currents, $\boldsymbol{J}_{i}$ and $\boldsymbol{M}_{i}$ over the two sides $(i=1,2)$ of the boundary surface around a dielectric object with the monopolar-RWG set $\left\{\boldsymbol{m}_{n}\right\}=\left\{\boldsymbol{f}_{n}^{1}, \boldsymbol{f}_{n}^{2}\right\}$ yields (see Fig. 1)

$$
\begin{aligned}
\boldsymbol{J}_{i} & \simeq \sum_{n=1}^{2 N_{e}} J_{n}^{i} \boldsymbol{m}_{n}=\sum_{n=1}^{N_{e}} a_{n}^{1, i} \boldsymbol{f}_{n}^{1}+\sum_{n=1}^{N_{e}} a_{n}^{2, i} \boldsymbol{f}_{n}^{2} \\
\boldsymbol{M}_{i} & \simeq \sum_{n=1}^{2 N_{e}} M_{n}^{i} \boldsymbol{m}_{n}=\sum_{n=1}^{N_{e}} b_{n}^{1, i} \boldsymbol{f}_{n}^{1}+\sum_{n=1}^{N_{e}} b_{n}^{2, i} \boldsymbol{f}_{n}^{2} \quad i=1,2
\end{aligned}
$$

where $N_{e}$ denotes the number of edges arising from the discretization of the boundary, and the sequences $\left\{J_{n}^{i}\right\}=\left\{a_{n}^{1, i}, a_{n}^{2, i}\right\},\left\{M_{n}^{i}\right\}=\left\{b_{n}^{1, i}, b_{n}^{2, i}\right\}$ represent the sets of unknown coefficients associated with the expansion of the electric and magnetic currents, respectively. The nonconforming monopolar-RWG set adopts the same local definition as the RWG set but with no continuity constraint across edges. The subsets $\left\{\boldsymbol{f}_{n}^{1}\right\}$ and $\left\{\boldsymbol{f}_{n}^{2}\right\}$ denote the monopolar-RWG contributions at the two facets that share the $n$th edge [6]. 


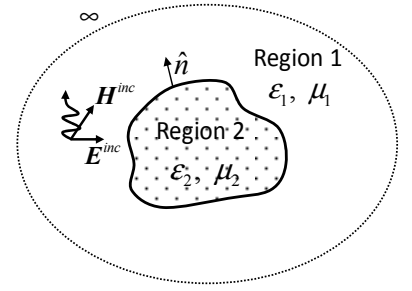

II

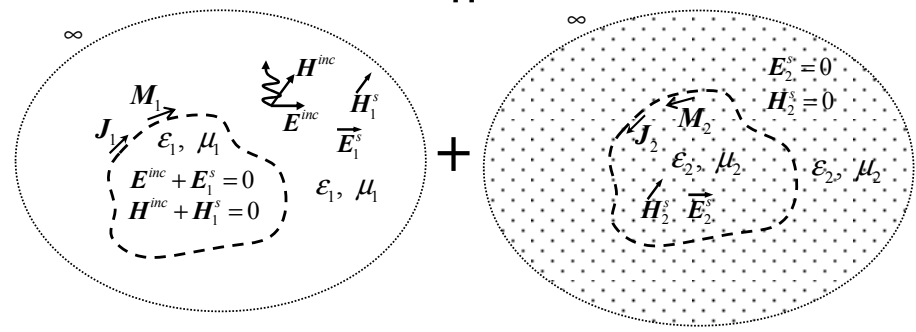

Fig. 1. Surface equivalence theorem for a penetrable object and plane wave excitation

The approximated scattered electric and magnetic fields, respectively, $\tilde{\boldsymbol{E}}_{i}^{s}$ and $\tilde{\boldsymbol{H}}_{i}^{s}$, obtained from the expanded currents in (1) and (2) are

$$
\begin{array}{rl}
\tilde{\boldsymbol{E}}_{i}^{s}=\sum_{n=1}^{2 N_{e}} \eta_{i} \boldsymbol{T}_{n}^{i} J_{n}^{i}-\sum_{n=1}^{2 N_{e}} \boldsymbol{K}_{n}^{i} M_{n}^{i} & i=1,2 \\
\tilde{\boldsymbol{H}}_{i}^{s}=\sum_{n=1}^{2 N_{e}} \boldsymbol{K}_{n}^{i} J_{n}^{i}+\sum_{n=1}^{2 N_{e}} \frac{1}{\eta_{i}} \boldsymbol{T}_{n}^{i} M_{n}^{i} & i=1,2
\end{array}
$$

where

$$
\begin{aligned}
& \boldsymbol{K}_{n}^{i}(\boldsymbol{r})=\nabla \times \iint_{\Gamma_{S}} G_{i}\left(\boldsymbol{r}, \boldsymbol{r}^{\prime}\right) \boldsymbol{m}_{n}\left(\boldsymbol{r}^{\prime}\right) d S^{\prime} \quad i=1,2 \\
& \boldsymbol{T}_{n}^{i}(\boldsymbol{r})=\frac{1}{j k_{i}}\left(\begin{array}{c}
\nabla \nabla \cdot \iint_{\Gamma_{S}} G_{i}\left(\boldsymbol{r}, \boldsymbol{r}^{\prime}\right) \boldsymbol{m}_{n}\left(\boldsymbol{r}^{\prime}\right) d S^{\prime} \\
+k_{i}^{2} \iint_{\Gamma_{S}} G_{i}\left(\boldsymbol{r}, \boldsymbol{r}^{\prime}\right) \boldsymbol{m}_{n}\left(\boldsymbol{r}^{\prime}\right) d S^{\prime}
\end{array}\right) \quad i=1,2
\end{aligned}
$$

The discretized PMCHWT is defined at the surface of the dielectric target in terms of the subtraction of the tangential components of the approximated fields in (3) and (4), so that

$$
\begin{aligned}
\left(\tilde{\boldsymbol{E}}_{1}^{s}-\tilde{\boldsymbol{E}}_{2}^{s}\right)_{\tan }= & \sum_{n=1}^{2 N_{e}}\left(\eta_{1} \boldsymbol{T}_{n}^{1}+\eta_{2} \boldsymbol{T}_{n}^{2}\right)_{\tan } J_{n} \\
& -\sum_{n=1}^{2 N_{e}}\left(\boldsymbol{K}_{n}^{1}+\boldsymbol{K}_{n}^{2}\right)_{\tan } M_{n}=-\boldsymbol{E}_{\tan }^{i n c} \\
\left(\tilde{\boldsymbol{H}}_{1}^{s}-\tilde{\boldsymbol{H}}_{2}^{s}\right)_{\tan }= & \sum_{n=1}^{2 N_{e}}\left(\boldsymbol{K}_{n}^{1}+\boldsymbol{K}_{n}^{2}\right)_{\tan } J_{n} \\
+ & \sum_{n=1}^{2 N_{e}}\left(\frac{1}{\eta_{1}} \boldsymbol{T}_{n}^{1}+\frac{1}{\eta_{2}} \boldsymbol{T}_{n}^{2}\right)_{\tan } M_{n}=-\boldsymbol{H}_{\mathrm{tan}}^{i n c}
\end{aligned}
$$

where $J_{n}=J_{n}^{1}=-J_{n}^{2}$ and $M_{n}=M_{n}^{1}=-M_{n}^{2}$. The quantities $k_{i}, \eta_{i}$ denote, respectively, the wave number and the impedance of the medium $i$. They are defined in terms of the free-space wave number $k_{0}$ and impedance $\eta_{0}$ as

$$
k_{i}=\sqrt{\varepsilon_{r, i}} k_{0}, \quad \eta_{r}=\eta_{0} / \sqrt{\varepsilon_{r, i}} \quad i=1,2
$$

where we assume different relative permittivities $\left(\varepsilon_{r, 1} \neq \varepsilon_{r, 2}\right)$ and same relative permeability $\left(\mu_{r, 1}=\mu_{r, 2}=1\right)$ in both regions. The Green's function of the medium $i$ is defined as $G_{i}=e^{-j k_{i} R} /(4 \pi R)$, where $R=\left|\boldsymbol{r}-\boldsymbol{r}^{\prime}\right|$. The vectors $\boldsymbol{E}_{\text {tan }}^{\text {inc }}$ and $\boldsymbol{H}_{\mathrm{tan}}^{\text {inc }}$ denote the tangential components of the incident electric and magnetic fields, respectively, at the meshed boundary surface $\Gamma_{S}$ (see Fig. 1).

The Galerkin testing of (7) and (8) results in the following expressions

$$
\begin{array}{r}
\iint_{S_{m}}\left(\tilde{\boldsymbol{E}}_{1}^{s}-\tilde{\boldsymbol{E}}_{2}^{s}\right) \cdot \boldsymbol{m}_{m}(\boldsymbol{r}) d S=-\iint_{S_{m}} \boldsymbol{E}^{i n c} \cdot \boldsymbol{m}_{m}(\boldsymbol{r}) d S \\
\iint_{S_{m}}\left(\tilde{\boldsymbol{H}}_{1}^{s}-\tilde{\boldsymbol{H}}_{2}^{s}\right) \cdot \boldsymbol{m}_{m}(\boldsymbol{r}) d S=-\iint_{S_{m}} \boldsymbol{H}^{i n c} \cdot \boldsymbol{m}_{m}(\boldsymbol{r}) d S \\
m=1 \ldots .2 N_{e}
\end{array}
$$

where the integral operator $\boldsymbol{K}_{n}^{i}$ is interpreted in the Cauchy principal value sense. The contribution of the $n$th monopolar-RWG basis function to the gradient of scalar potentials (electric or magnetic) $\nabla \Phi_{n}^{i}$ at each medium $(i=1,2)$ yields $[6]$

$$
\begin{aligned}
\nabla \Phi_{n}^{i}(\boldsymbol{r}) & =j \frac{\eta_{i}}{k_{i}} \iint_{S_{n}} \nabla G_{i} \nabla^{\prime} \cdot \boldsymbol{m}_{n}\left(\boldsymbol{r}^{\prime}\right) d S^{\prime} \\
& -j \frac{\eta_{i}}{k_{i}} \oint_{\partial S_{n}} \nabla G_{i}\left(\boldsymbol{m}_{n}\left(\boldsymbol{r}^{\prime}\right) \cdot \hat{n}_{c}^{n}\right) d L^{\prime}
\end{aligned}
$$

where $\partial S_{n}$ and $\hat{n}_{c}^{n}$ denote, respectively, the closed contour around the source triangle $S_{n}$ and the unit vector perpendicular to this contour. The testing integrals in (10) become unbounded because the line integral in (11) becomes non-integrable when the field point approaches the source point. In this work we circumvent this problem through non-Galerkin testings in the vicinity of the boundary inside the region where the fields must be null in accordance with the equivalence theorem.

\section{A. Volumetric testing}

The testing of the fields is carried out over a set of tetrahedral elements attached to the boundary surface (see Figs. 1, 2) [6]. The monopolar-RWG discretization of the PMCHWT formulation with volumetric testing thus leads to the following matrix system 


$$
\begin{array}{r}
\iiint_{V_{m}^{2}} \tilde{\boldsymbol{E}}_{1}^{s} \cdot \boldsymbol{v}_{m}^{2}(\boldsymbol{r}) d V-\iiint_{V_{m}^{1}} \tilde{\boldsymbol{E}}_{2}^{s} \cdot \boldsymbol{v}_{m}^{1}(\boldsymbol{r}) d V=-\iiint_{V_{m}^{2}} \boldsymbol{E}^{i n c} \cdot \boldsymbol{v}_{m}^{2}(\boldsymbol{r}) d V \\
\iiint_{V_{m}^{2}} \tilde{\boldsymbol{H}}_{1}^{s} \cdot \boldsymbol{v}_{m}^{2}(\boldsymbol{r}) d V-\iiint_{V_{m}^{1}} \tilde{\boldsymbol{H}}_{2}^{s} \cdot \boldsymbol{v}_{m}^{1}(\boldsymbol{r}) d V=-\iiint_{V_{m}^{2}} \boldsymbol{H}^{i n c} \cdot \boldsymbol{v}_{m}^{2}(\boldsymbol{r}) d V \\
m=1 \ldots .2 N_{e}
\end{array}
$$

where $V_{m}^{i}$ denotes the $m$-th tetrahedral testing element attached to the boundary surface, in the medium $i$, where the monopolar-SWG testing functions $\left\{\boldsymbol{v}_{m}^{i}\right\}$ are defined (see Fig. 2). This testing set, defined in an analogous manner as the monopolar-RWG set, adopts the definition of SWG basis functions [9] in each tetrahedron but do not set any continuity constraints across common faces. The $n$th monopolar-SWG testing function shares the face at the surface where the corresponding $n$th monopolar-RWG function is defined. In view of Fig. 2, the testing elements are attached to the boundary in the side where, for that particular region, in accordance with the equivalence theorem, the fields must be zero. The accuracy of this implementation depends on the height of the tetrahedral elements $(H)$, which we define, in view of Fig. 2, with the same value at both regions, as a fraction of the lengths $(h)$ of the associated edges in the matching triangles.

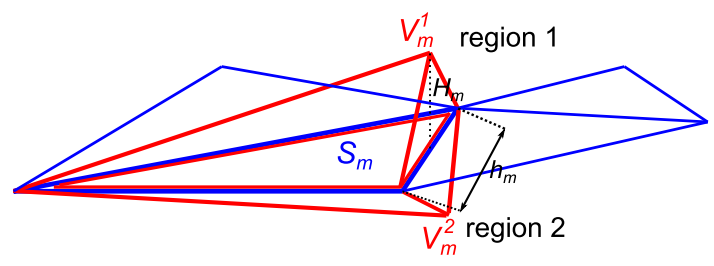

Fig. 2. Volumetric testing over the tetrahedral elements $V_{m}^{1}$ and $V_{m}^{2}$, attached to the triangular facet $S_{m}$, and defined, respectively, inside regions 1 and 2. The height of the $m$ th testing tetrahedral element $H_{m}$ is defined as a fraction of $h_{m}$, the length of the associated edge in $S_{m}$

\section{B. Tangential-normal testing}

The fields are tested with RWG basis functions embracing a pair of connected triangles such that one triangle matches one facet arising in the surface triangulation and the other one is orientated normally to the boundary, inside the region where the null field condition needs to be accomplished (see Fig. 3).

The monopolar-RWG discretization of the PMCHWT formulation with tangential-normal testing gives rise to

$$
\begin{array}{r}
\iint_{S_{m} \cup S_{m}^{2}} \tilde{\boldsymbol{E}}_{1}^{s} \cdot \boldsymbol{t}_{m}^{2}(\boldsymbol{r}) d S-\iint_{S_{m} \cup S_{m}^{1}} \tilde{\boldsymbol{E}}_{2}^{s} \cdot \boldsymbol{t}_{m}^{1}(\boldsymbol{r}) d S=-\iint_{S_{m} \cup S_{m}^{2}} \boldsymbol{E}^{i n c} \cdot \boldsymbol{t}_{m}^{2}(\boldsymbol{r}) d S \\
\iint_{S_{m} \cup S_{m}^{2}} \tilde{\boldsymbol{H}}_{1}^{s} \cdot \boldsymbol{t}_{m}^{2}(\boldsymbol{r}) d S-\iint_{S_{m} \cup S_{m}^{1}} \tilde{\boldsymbol{H}}_{2}^{s} \cdot \boldsymbol{t}_{m}^{1}(\boldsymbol{r}) d S=-\iint_{S_{m} \cup S_{m}^{2}} \boldsymbol{H}^{i n c} \cdot \boldsymbol{t}_{m}^{2}(\boldsymbol{r}) d S \\
m=1 \ldots .2 N_{e}
\end{array}
$$

where $S_{m} \cup S_{m}^{i}$ denotes the $m$-th domain in a particular region $(i=1,2)$, where the corresponding $\mathrm{RWG}$ testing functions $\left\{\boldsymbol{t}_{m}^{i}\right\}$ are defined. The testing of $\nabla \Phi_{n}^{i}$ with tangential-normal testing functions results in

$$
\iint_{S_{m} \cup S_{m}^{i}} \boldsymbol{t}_{m}^{i} \cdot \nabla \Phi_{n}^{i}(\boldsymbol{r}) d S=-\iint_{S_{m} \cup S_{m}^{i}} \Phi_{n}^{i}(\boldsymbol{r}) \nabla \cdot \boldsymbol{t}_{m}^{i} d S
$$

because $\iint_{S_{m} \cup S_{m}^{i}} \nabla \cdot\left(\boldsymbol{t}_{m}^{i} \Phi_{n}^{i}(\boldsymbol{r})\right) d S=0$ thanks to the normal continuity of the tangential-normal testing functions across the edge $m$. The Kernel hypersingular contributions in (11) are now canceled out and the computational burden is reduced when compared with the volumetric testing [8].

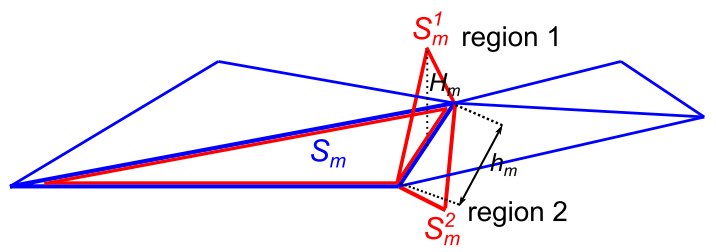

Fig. 3. Tangential-normal testing with RWG functions defined over the pairs of triangles $S_{m}$ and $S_{m}^{1}$, inside region 1 , or $S_{m}$ and $S_{m}^{2}$, inside region 2 . The height of the triangles oriented normally with respect to the boundary, $S_{m}^{1}$ and $S_{m}^{2}, H_{m}$, is defined as a fraction of $h_{m}$, the length of the associated edge in $S_{m}$

\section{NUMERICAL RESULTS}

The testing object in Fig. 4 is a low-contrast dielectric sphere with diameter $0.2 \mathrm{~m} \quad\left(\varepsilon_{\mathrm{r}}=2\right)$, meshed with 128 triangular facets. In Fig. 5, we test a high-contrast dielectric cube with side $0.1 \mathrm{~m}\left(\varepsilon_{\mathrm{r}}=100\right)$ meshed with 192 triangles. Both objects are immersed in the free space. We show RCS results computed with the monopolar-RWG discretization of the PMCHWT formulation with tangential-normal testing (PMCHWT[tn-monoRWG]) and volumetric testing (PMCHWT[V-monoRWG]). These are compared against the RWG-discretization of the PMCHWT (PMCHWT[RWG]) and reference results. Whereas for the sphere, the Mie series solution can be adopted as reference, for the cube, with no analytical solution available, we adopt the result computed with PMCHWT[RWG] and a very dense mesh (32400 unknowns). Note that the observation angle of $0^{\circ}$ in Figs. 4 and 5 corresponds with the backscattering direction. In both examples the targets are impinged by an $x$-polarized $+z$-propagating plane wave, the free-space wavelength $\left(\lambda_{0}\right)$ is set to $1 \mathrm{~m}$ and the height of the testing elements, $H$, is set to be $h / 10$. In view of Figs. 4 and 5 , very good agreement is observed between the RCS computed with the several implementations. Moreover, in view of Fig. 5, for the case of the target with sharp edges and corners, the RCS computed with nonconforming 
PMCHWT implementations shows even better accuracy than the RCS computed with the divergence-conforming RWG implementation and same degree of meshing. We attribute this improvement to the better modeling with the nonconforming schemes of the singular currents arising in the vicinity of sharp edges.

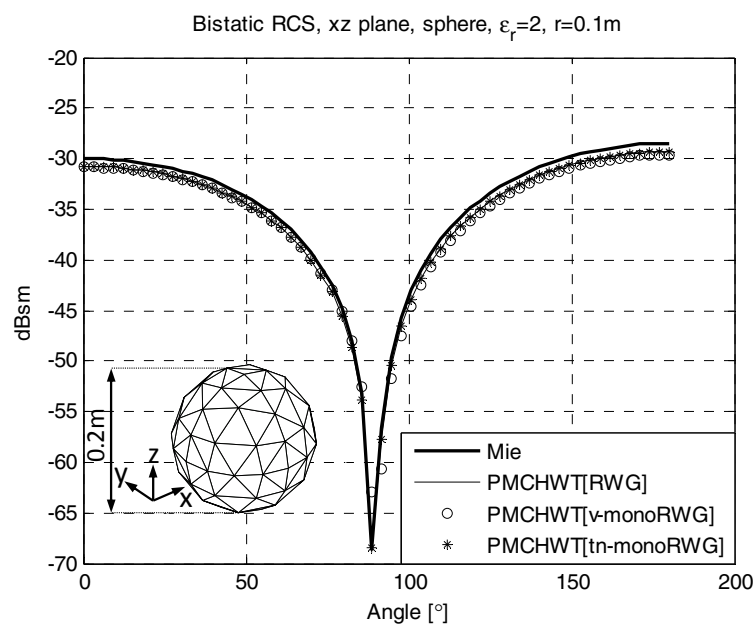

Fig. 4. xz plane cut of the bistatic RCS computed with PMCHWT[vmonoRWG], PMCHWT[tn-monoRWG] and PMCHWT[RWG] for a dielectric sphere with diameter $0.2 \mathrm{~m}$ and $\varepsilon_{\mathrm{r}}=2$, meshed with 128 triangles,. The impinging plane wave is $\mathrm{x}$-polarized travelling in the $+\mathrm{z}$-direction and $\lambda_{0}=1 \mathrm{~m}$. Mie series is adopted as reference.

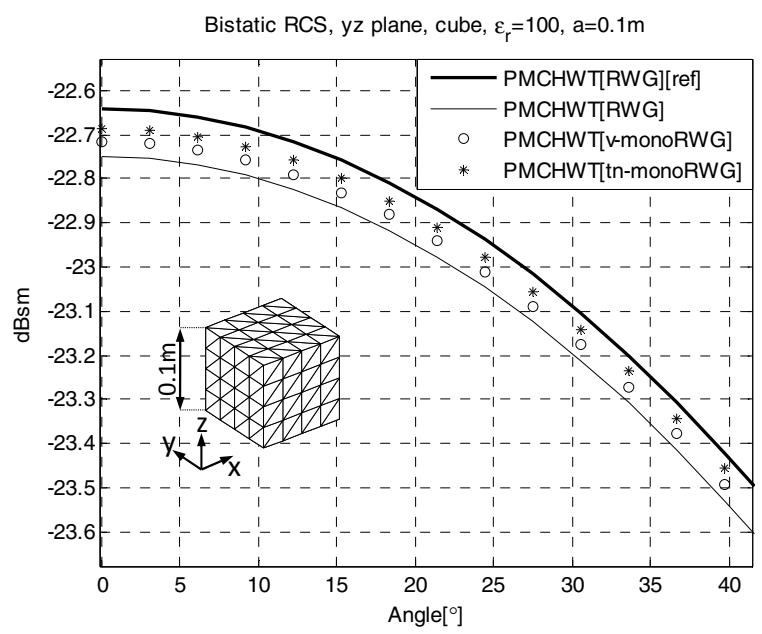

Fig. 5. yz plane cut of the bistatic RCS near the backward direction computed with PMCHWT[v-monoRWG], PMCHWT[tnmonoRWG] and PMCHWT[RWG] for a dielectric cube with side $0.1 \mathrm{~m}$ and $\varepsilon_{\mathrm{r}}=100$, meshed with 192 triangles. The impinging wave is $\mathrm{x}$-polarized travelling in $+\mathrm{z}$-direction and $\lambda_{0}=1 \mathrm{~m}$. The result computed with PMCHWT[RWG] on very fine mesh is adopted as reference.

\section{CONCLUSION}

We present a nonconforming discretization of the PMCHWT integral formulation, where the unknown currents are expanded with the nonconforming monopolarRWG set, which does not impose normal continuity across the edges arising from the discretization. The hypersingular Kernel contributions arising in the nonconforming discretization of scalar potentials, electric and magnetic, are tackled by two different non-Galerkin testing strategies, volumetric or tangential-normal. Very good agreement is observed in the computed RCS between the conventional divergence-conforming RWG-discretization of the PMCHWT and the new nonconforming monopolar-RWG discretizations for a particular meshing of a homogeneous dielectric target, either smooth or sharp-edged. In addition, improved performance can be observed in the RCS-analysis of a cube with high contrast. We attribute this to the better modelling with nonconforming approaches of the singular fields near sharp edges. Since nonconforming schemes undertake the expansion of the boundary unknowns over facets, with no continuity constraint across edges, these monopolar-RWG PMCHWT implementations appear as promising tools for the robust and agile analysis of composite objects.

\section{ACKNOWLEDGMENT}

This work has been supported by the Spanish "Comisión Interministerial de Ciencia y Tecnologíia" (CICYT) under projects TEC2013-47360-C3-1-P and TEC2016-78028-C31-P.

\section{REFERENCES}

[1] A. J. Poggio and E. K. Miller, "Integral equation solutions of threedimensional scattering problems," in Computer Techniques for Electromagnetics, R. Mittra, Ed. Oxford, UK: Pergamon Press, 1973, ch. 4.

[2] T. K. Wu and L. L. Tsai, "Scattering from arbitrarily-shaped lossy dielectric bodies of revolution," Radio Science, vol. 12, pp. 709-718, Sep.-Oct. 1977.

[3] Y. Chang and R. F. Harrington, "A surface formulation for characteristic modes of material bodies," IEEE Transactions on Antennas and Propagation, vol. AP-25, pp. 789-795, Nov. 1977.

[4] S. M. Rao, D. R. Wilton and A. W. Glisson, "Electromagnetic Scattering by surfaces of Arbitrary Shape," IEEE Transactions on Antennas and Propagation, vol. AP-30, No. 3, pp. 409-418, May 1982.

[5] P. Ylä-Oijala, M. Taskinen, and J. Sarvas, "Surface integral equation method for general composite metallic and dielectric structures with junctions," Progress In Electromagnetics Research, Vol. 52, pp. 81$108,2005$.

[6] E. Ubeda, Juan M. Rius and A. Heldring, "Nonconforming discretization of the Electric-Field Integral Equation for closed perfectly conducting objects," IEEE Transactions on Antennas and Propagation, vol. 62, no. 8, pp. 4171-4186, August 2014.

[7] E. Ubeda, Juan M. Rius, A. Heldring and I. Sekulic, "Volumetric testing parallel to the boundary surface for a nonconforming discretization of the Electric-Field Integral Equation," IEEE Transactions on Antennas and Propagation, vol. 63, no. 7, pp. 32863291, July 2015.

[8] E. Ubeda, I. Sekulic, J. M. Rius, A. Heldring, "Tangential-normal surface testing for the nonconforming discretization of the ElectricField Integral Equation," IEEE Antennas and Wireless Propagation Letters, vol. 15, 2016, pp.1581-1584.

[9] D. H. Schaubert, D. R. Wilton and A. W. Glisson, “A Tetrahedral Modeling for Electromagnetic Scattering by arbitrarily shaped Inhomogeneous Dielectric Bodies," IEEE Transactions on Antennas and Propagation, vol. AP-32, No. 1, January 1984. 